\title{
Algèbre Homologique des $N$-Complexes et Homologie de Hochschild aux Racines de l'Unité
}

\author{
par \\ Christian KASSEL * et Marc WAMBST*
}

\begin{abstract}
We set up a homological algebra for $N$-complexes, which are graded modules together with a degree -1 endomorphism $d$ satisfying $d^{N}=0$. We define Tor- and Ext-groups for $N$-complexes and we compute them in terms of their classical counterparts $(N=2)$ As an application, we get an alternative definition of the Hochschild homology of an associative algebra out of an $N$-complex whose differential is based on a primitıve $N$-th root of unity.
\end{abstract}

\section{Résumé}

Nous développons une algèbre homologıque pour les $N$-complexes, c'est-à-dire pour des nıodules gradues munis d'un endomorphisme $d$ de degre -1 tel que $d^{N}=0$. Dans ce cadre nous définissons des groupes Tor et Ext que nous calculons en fonction des groupes Tor et Ext classiques $(N=2)$. Comme application, nous obtenons l'homologie de Hochschild d'une algèbre associative comme l'homologie d'un $N$-complexe dont la dıfférentielle s'exprıme à l'aide d'une racine prımitıve $N$-ıème de l'unité.

\section{Introduction}

Le point de départ de ce travail est l'observation que l'homologie de Hochschild d'une algèbre associative $A$ au-dessus d'un anneau commutatif $k$ peut se définir à partir de différentielles dans lesquelles -1 est remplacé par n'importe quelle racine de l'unité.

Pour tout $n \in \mathbb{Z}$, posons $C_{n}(A)=A^{\otimes(n+1)}$ si $n \geq 0$ et $C_{n}(A)=0$ si $n<0$. Définissons $b: C_{n}(A) \rightarrow C_{n-1}(A)$ par

Communıqué par M. Kashıwara le 8 maı 1997.

1991 Mathematics Subject Classifications: 18G25, 18G30, 18G35, 05A30, 81R50

* Institut de Recherche Mathématique Avancée, Université Louss Pasteur-C.N.R.S., 7 rue René Descartes. 67084 Strasbourg Cedex. France. 


$$
b\left(a_{0} \otimes a_{1} \otimes \cdots \otimes a_{n}\right)=\sum_{i=0}^{n-1} q^{i} a_{0} \otimes \cdots \otimes a_{i} a_{i+1} \otimes \cdots \otimes a_{n}+q^{n} a_{n} a_{0} \otimes a_{1} \otimes \cdots \otimes a_{n-1}
$$

où $q$ est un scalaire et $a_{0}, a_{1}, \cdots, a_{n} \in A$. Fixons maintenant un entier $N \geq 2$. On vérifie que $b^{N}=0$ dans les deux cas suivants:

(i) $q$ est une racine $N$-ième de 1 différente de 1 ;

(ii) $q=1$ et $N=0$ dans l'algèbre $A$.

Lorsque $q=-1$ et $N=2$, l'application $b$ est le bord de Hochschild standard et l'homologie du complexe $(C(A), b)$ est l'homologie de Hochschild $H H_{*}(A)$ de l'algèbre $A$. Lorsque $N>2$, le couple $(C(A), b)$ n'est plus un complexe de chaînes. On peut cependant définir des avatars ${ }_{p} H H_{*}(A)$ de l'homologie de Hochschild pour $p=1, \cdots, N-1$ par

$$
{ }_{p} H H_{n}(A)=\frac{\operatorname{Ker}\left(b^{p}: C_{n}(A) \rightarrow C_{n-p}(A)\right)}{\operatorname{Im}\left(b^{N-p}: C_{n+N-p}(A) \rightarrow C_{n}(A)\right)} .
$$

Notre premier résultat (dont une version cohomologique a été annoncée par Dubois-Violette [1]) énonce que, sous certaines conditions, les groupes ainsi définis sont soit nuls, soit isomorphes aux groupes de Hochschild usuels, ce qui nous donne une définition alternative et exotique de l'homologie de Hochschild.

Théorème 1. Considérons un entier $N \geq 2$, un scalaire $q$ et une algèbre associative unifère $A$. Sous chacune des deux hypothèses suivantes:

(a) $q \neq 1$ est une racine primitive $N$-ième de l'unité,

(b) $N$ est un nombre premier, $q=1$ et $A$ est une $\mathbb{Z} / N$-algèbre. nous avons, pour tout entier $n \geq 0$ et tout $p=1, \cdots, N-1$,

$$
{ }_{p} H H_{n}(A) \cong\left\{\begin{array}{cl}
H H_{2(n-p+1) / N}(A) & \text { si } n+1 \equiv p \bmod N \\
H H_{(2 n+2-N) / N}(A) & \text { si } n+1 \equiv 0 \bmod N \\
0 & \text { dans tous les autres cas. }
\end{array}\right.
$$

Pour établir ce théorème, nous nous plaçons dans le cadre général des $N$-complexes. c'est-à-dire des modules gradués munis d'un endomorphisme $d$ de degré -1 tel que $d^{N}=0$. Ces objets ont été abondamment utilisés dans les travaux récents de Dubois-Violette et al. et de Kapranov sur le calcul différentiel quantique (cf. [1], [2], [3], [5]). Un embryon d'algèbre homologique adaptée aux $N$-complexes est apparu dans [5].

Dans cet article, nous avons développé systématiquement une telle algèbre homologique en définissant la notion de $N$-résolution projective ou injective ainsi que des groupes ${ }_{p}$ Tor et ${ }_{p}$ Ext qui jouissent de propriétés parallèles aux groupes Tor et Ext classiques. Le cadre dans lequel nous travaillons est celui de 
l'algèbre homologique relative d'Eilenberg et Moore. Entre les groupes ${ }_{p}$ Tor et ${ }_{p}$ Ext et leurs pendants classiques, nous établissons des isomorphismes formellement similaires à ceux du théorème 1 .

Ce dernier est alors conséquence de l'isomorphisme

$$
{ }_{p} H H_{*}(A, A) \cong{ }_{p} \operatorname{Tor}_{*}^{A \otimes A^{\circ}}(A, A)
$$

établi au $\$ 5$ au moyen d'une $N$-résolution projective (relativement aux suites exactes de $A$-bimodules munies d'un scindage $k$-linéaire). Pour démontrer l'acyclicité de cette résolution. nous construisons une homotopie à l'aide d'identités remarquables dans l'algèbre des opérateurs aux $q$-différences engendrée par deux indéterminées $X$ et $Y$ soumises à la relation $Y X-q X Y=1$. Ces identités sont valides précisément sous les mêmes hypothèses sur le scalaire $q$ que celles du théorème 1 .

Pour terminer, signalons que des différentielles analogues à celles de (0.1) et des groupes d'homologie du type $(0.2)$ ont été considérés dès les années 1940. C'est ainsi que Mayer [6] a introduit des groupes d'homologie singulière des espaces topologiques à coefficients dans un corps fini $\mathbb{Z} / \ell$ avec une différentielle du type $(0.1)$ dans laquelle $q=1$. Spanier [9] a démontré que les groupes d'homologie de Mayer se réduisaient aux groupes d'homologie traditionnels dans un théorème dont l'énoncé est formellement le même que celui du théorème 1 plus haut. Pour $q \neq 1$ il y a des résultats similaires de Sarkaria [7]. [8] concernant l'homologie simpliciale.

Nous remercions chaleureusement Michel Dubois-Violette pour ses remarques et ses encouragements.

\section{Conventions et Notations}

Dans toute la suite, on fixe un entier $N \geq 2$ et un anneau commutatif $k$ qu'on appellera l'anneau de base. Toutes les opérations d'algèbre linéaire effectuées le sont dans la catégorie des $k$-modules. Les $k$-algèbres considérées dans ce travail sont associatives et unifères.

On choisit un élément $q$ de $k$. Pour tout $n \in \mathbb{Z}$, nous posons

$$
[n]=\left\{\begin{array}{ccc}
\frac{q^{n}-1}{q-1} & \text { si } & q \neq 1 \\
n & \text { si } & q=1
\end{array}\right.
$$

Si $r \in \mathbb{N}$ on convient que $[0] !=1$ et $[r] !=[1][2] \cdots[r]$ si $r>0$. Enfin, pour $0 \leq r, s$ $\leq N-1$ on définit les $q$-coefficients binomiaux $(r, s)$ par

$$
(r, s)=\frac{[r+s] !}{[r] ![s] !}
$$




\section{\$1. Généralités sur les $\mathbb{N}$-Complexes et leur Homologie}

Nous nous plaçons dans une catégorie abélienne $\mathscr{A}$.

1.1. Définitions. Un $N$-complexe est un objet $\mathbb{Z}$-gradué $C$ de $\not 2$ muni d'un endomorphisme $d$ de degré -1 tel que $d^{N}=0$. On notera $(C, d)$ la donnée d'un $N$-complexe. On dit que $(C, d)$ est positif (resp. négatif) si $C_{n}=0$ pour tout $n<0$ (resp. pour tout $n>0$ ).

Un morphisme $f:(C, d) \rightarrow\left(C^{\prime}, d^{\prime}\right)$ de $N$-complexes est un morphisme $f: C \rightarrow C^{\prime}$ d'objets $\mathbb{Z}$-gradués de $\not{A l}$ qui est de degré 0 et tel que $d^{\prime} f=f d$.

Deux morphismes $f, g:(C, d) \rightarrow\left(C^{\prime}, d^{\prime}\right)$ de $N$-complexes sont homotopes s'il existe un morphisme $h: C \rightarrow C^{\prime}$ de degré $N-1$ tel que

$$
f-g=\sum_{\imath=0}^{N-1} d^{N-1-t} h d^{\imath}
$$

L'homotopie est une relation d'équivalence.

1.2. Homologie. A tout $N$-complexe $(C, d)$ et tout $p=1, \ldots, N-1$ on peut associer les groupes d'homologie ${ }_{p} H_{*}(C)$ définis par

$$
{ }_{p} H_{n}(C)=\frac{\operatorname{Ker}\left(d^{p}: C_{n} \rightarrow C_{n-p}\right)}{\operatorname{Im}\left(d^{N-p}: C_{n+N-p} \rightarrow C_{n}\right)} .
$$

Ces groupes sont fonctoriels. c'est-à-dire tout morphisme $f:(C, d) \rightarrow\left(C^{\prime}, d^{\prime}\right)$ de $N$-complexes induit une application $f_{*}:{ }_{p} H_{*}(C) \rightarrow{ }_{p} H_{*}\left(C^{\prime}\right)$ telle que id ${ }_{*}$ soit l'identité et $(f \circ g)_{*}=f_{*} \circ g_{*}$ (lorsque $f$ et $g$ sont composables).

1.3. Lemme. Deux morphismes homotopes $f . g: C \rightarrow C^{\prime}$ de $N$-complexes induisent la même application sur les groupes d'homologie.

Démonstration. Si $x \in C_{n}$ tel que $d^{p} x=0$ représente un élément de ${ }_{p} H_{n}(C)$. alors nous tirons de (1.1) que

$$
f(x)-g(x)=\sum_{i=0}^{p-1} d^{N-1-\imath} h d^{i}(x)=d^{N-p}\left(\sum_{i=0}^{p-1} d^{p-1-\imath} h d^{i}(x)\right)
$$

ce qui montre que $f$ et $g$ induisent la même application ${ }_{p} H_{n}(C) \rightarrow{ }_{p} H_{n}\left(C^{\prime}\right)$.

1.4. Acychicité. On dit que le $N$-complexe $(C, d)$ est acyclique si ${ }_{p} H_{n}(C)$ $=0$ pour tout $n \in \mathbb{Z}$ et tout $p=1, \cdots, N-1$. Kapranov a montré que pour que $(C$, d) soit acyclique, il suffit qu'il existe $p$ tel que ${ }_{p} H_{n}(C)=0$ pour tout $n \in \mathbb{Z}$ (voir [5], Proposition 1.5). 
1.5. Les morphismes $i_{*}$ et $d_{*}$. Soit $(C, d)$ un $N$-complexe. Lorsque $N \geq$ 3 , on a deux familles importantes de morphismes naturels

$$
i_{*}:{ }_{p} H_{\imath}(C) \rightarrow{ }_{p+1} H_{i}(C) \text { et } \quad d *:{ }_{p} H_{i}(C) \rightarrow{ }_{p-1} H_{\imath-1}(C) .
$$

La première. de degré 0 , est induite par l'inclusion $\operatorname{Ker}\left(d^{p}\right) \subset \operatorname{Ker}\left(d^{p+1}\right)$. La seconde, de degré -1 , est induite $\operatorname{par} d: \operatorname{Ker}\left(d^{p-1}\right) \rightarrow \operatorname{Ker}\left(d^{p-1}\right)$. On a le résultat suivant dont on trouvera une démonstration dans [2], Lemma 1.

1.6. Lemme. Pour tous les entiers strictement positifs $r$. $p$ tels que $0<p+r$ $<N$, il existe une longue suite exacte naturelle de la forme

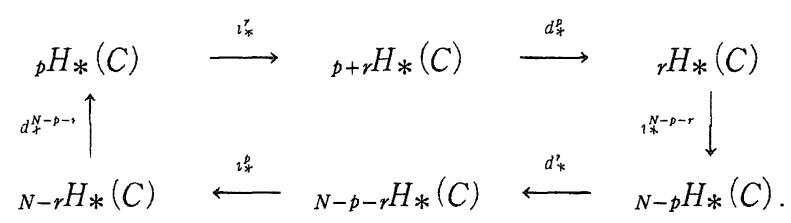

1.7. Définition. Use suite exacte courte $0 \rightarrow C^{\prime} \stackrel{u}{\rightarrow} C \stackrel{v}{\rightarrow} C^{\prime \prime} \rightarrow 0$ de $N$-complexes est la donnée de morphismes $C^{\prime} \stackrel{u}{\rightarrow} C \stackrel{v}{\rightarrow} C^{\prime \prime}$ de $N$-complexes tels que tout $n \in \mathbb{Z}$ la suite $0 \rightarrow C_{n}{ }^{\prime} \stackrel{u}{\rightarrow} C_{n} \stackrel{v}{\rightarrow} C^{\prime \prime}{ }_{n} \rightarrow 0$ est exacte.

1.8. Lemme. Soit $0 \rightarrow C^{\prime} \stackrel{u}{\rightarrow} C \stackrel{v}{\rightarrow} C^{\prime \prime} \rightarrow 0$ une suite exacte courte de $N$-complexes. Alors il existe une longue suite exacte naturelle

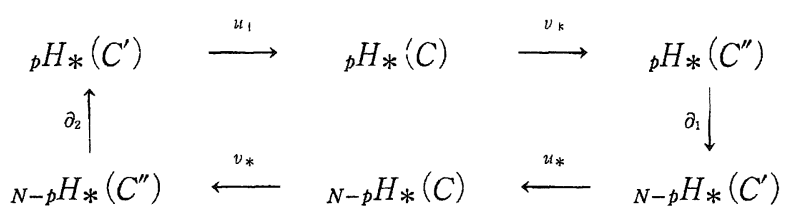

où $\partial_{1}$ est une application de degré $-p$ et $\partial_{2}$ une application de degré $-(N-p)$.

Démonstration. Appliquons le lemme du serpent au diagramme de suites exactes

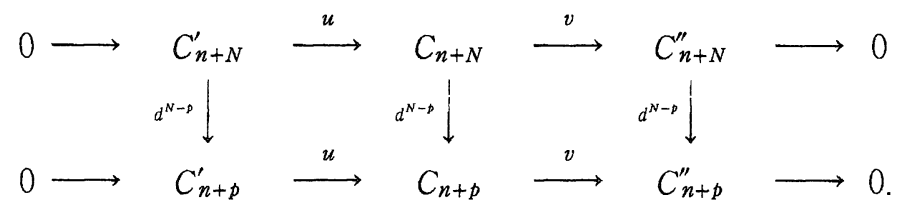

Nous obtenons pour tout $n$ les deux suites exactes

$$
0 \longrightarrow \operatorname{Ker}\left(d^{N-p}\right) \cap C_{n}^{\prime} \stackrel{u}{\longrightarrow} \operatorname{Ker}\left(d^{N-p}\right) \cap C_{n} \stackrel{v}{\longrightarrow} \operatorname{Ker}\left(d^{N-p}\right) \cap C_{n}^{\prime \prime}
$$


et

$$
C_{n+p}^{\prime} / \operatorname{Im}\left(d^{N-p}\right) \stackrel{u}{\longrightarrow} C_{n+p} / \operatorname{Im}\left(d^{N-p}\right) \stackrel{v}{\longrightarrow} C_{n+p}^{\prime \prime} / \operatorname{Im}\left(d^{N-p}\right) \longrightarrow 0,
$$

que nous assemblons dans le diagramme commutatif de suites exactes

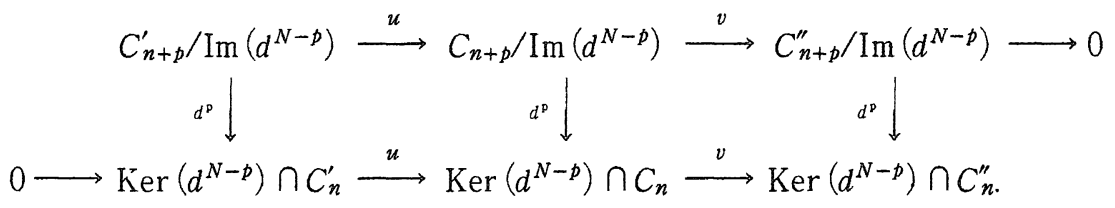

Une autre application du lemme du serpent nous donne la longue suite exacte désirée.

\section{§2. Résolutions}

On se place à nouveau dans une catégorie abélienne $\mathscr{A}$.

2.1. Rappels d'algèbre homologique relative (voir [4]). Une classe projective dans $\mathscr{A}$ est un couple $(\mathscr{P}, \mathscr{S})$ où $\mathscr{P}$ est une classe d'objets de $\mathscr{A}$ et $\mathscr{S}$ est une classe de suites $Y^{\prime} \stackrel{u}{\rightarrow} Y \stackrel{v}{\rightarrow} Y^{\prime \prime}$ de $\mathscr{A}$ telles que $v u=0$, verifiant les trois axiomes suivants:

(i) Un objet $P$ de $\mathscr{A}$ est dans $\mathscr{P}$ si et seulement si pour toute suite $Y^{\prime} \stackrel{u}{\rightarrow} Y$ $\stackrel{v}{\rightarrow} Y^{\prime \prime}$ de $\mathscr{S}$, la suite de groupes abéliens

$$
\operatorname{Hom}\left(P . Y^{\prime}\right) \rightarrow \operatorname{Hom}(P, Y) \rightarrow \operatorname{Hom}\left(P, Y^{\prime \prime}\right)
$$

est exacte.

(ii) Une suite $Y^{\prime} \stackrel{u}{\rightarrow} Y \stackrel{v}{\rightarrow} Y^{\prime \prime}$ de $\not A$ est dans $\&$ si et seulement si pour tout objet $P$ de $\mathscr{P}$, la suite

$$
\operatorname{Hom}\left(P, Y^{\prime}\right) \rightarrow \operatorname{Hom}(P, Y) \rightarrow \operatorname{Hom}\left(P, Y^{\prime \prime}\right)
$$

est exacte.

(iii) Pour tout morphisme $X \rightarrow Y$ de $\mathscr{A}$ il existe un objet $P$ de $\mathscr{P}$ et un morphisme $P \rightarrow X$ de $\mathscr{A}$ tels que $P \rightarrow X \rightarrow Y$ est dans $\mathscr{\&}$.

Dans une classe projective $(\mathscr{P}, \mathscr{S})$, la classe $\mathscr{P}$ d'objets et la classe $\&$ de suites se déterminent mutuellement. Les objets de $\mathscr{P}$ sont appelés $\mathscr{P}$-projectifs.

2.2. Exemples. (a) (Classe projective absolue) C'est la classe projective

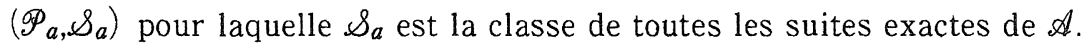

(b) (Classe projective $k$-scindée) Supposons que la catégorie $\mathscr{A}$ soit $k$-linéaire au-dessus de l'anneau de base $k$. Il existe alors une classe projective 
$\left(\mathscr{P}_{k}, \mathscr{\bigotimes}_{k}\right)$ pour laquelle $\mathscr{\&}_{k}$ est constituée de toutes les suites $Y^{\prime} \stackrel{u}{\rightarrow} Y \stackrel{v}{\rightarrow} Y^{\prime \prime}$ telles que le morphisme induit $Y^{\prime} \rightarrow \operatorname{Ker}(v)$ est un épimorphisme $k$-scindé, c'est-à-dire possédant un inverse à droite qui est $k$-linéaire.

Par exemple, si $\mathscr{A}$ est la catégorie des modules à gauche sur une $k$-algèbre $A$, alors $\mathscr{P}_{k}$ est formée de tous les $A$-modules de la forme $A \otimes_{k} V$ où $V$ est un $k$-module quelconque.

2.3. Définitions. On fixe une classe projective $(\mathscr{P}, \mathscr{S})$ de $\mathscr{A}$. Un $N^{-}$ complexe $(C, d)$ est $\mathscr{P}$-exact si le $N$-complexe induit $\operatorname{Hom}(P, C)$ est acyclique pour tout objet $\mathscr{P}$-projectif $P$.

Soit $L$ un objet de la catégorie $\mathscr{A}$. Une $N$-résolution $\mathscr{P}$-projective de $L$ est la donnée d'un $N$-complexe positif $(P, d)$ de $\mathscr{A}$ et d'un morphisme $\varepsilon: P_{0} \rightarrow L$ tels que

(i) l'objet $P_{n}$ est $\mathscr{P}$-projectif pour tout $n \geq 0$,

(ii) la suite

$$
\stackrel{\stackrel{d}{\longrightarrow}}{\longrightarrow} P_{2} \stackrel{d}{\longrightarrow} P_{1} \stackrel{d}{\longrightarrow} P_{0} \stackrel{\varepsilon}{\longrightarrow} L \longrightarrow 0
$$

est un $N$-complexe $\mathscr{P}$-exact.

Nous noterons $\left(P, d, \varepsilon: P_{0} \rightarrow L\right)$ la donnée d'une $N$-résolution $\mathscr{P}$-projective de $L$.

2.4. Existence de $N$-résolutions $\mathscr{P}$-projectives. Démontrons que tout objet $L$ de $\mathscr{A}$ possède une $N$-résolution $\mathscr{P}$-projective. On sait que $L$ a une résolution $\mathscr{P}$-projective au sens de l'algèbre homologique traditionnelle. Choisissons-en une, soit $(Q, \delta) \stackrel{\varepsilon}{\rightarrow} L$. Considérons la suite d'applications

$$
\cdots \stackrel{\text { Id }}{\longrightarrow} Q_{2} \stackrel{\delta}{\longrightarrow} Q_{1} \stackrel{\delta}{\longrightarrow} Q_{0} \stackrel{\text { id }}{\longrightarrow} \cdots \stackrel{\text { td }}{\longrightarrow} Q_{0} \stackrel{\varepsilon}{\longrightarrow} L \longrightarrow 0
$$

où l'application identité a été insérée $N-2$ fois à la place de tous les objects $Q_{2}$ $(i \geq 0)$. On vérifie facilement que le $N$-complexe (2.1) est une $N$-résolution $\mathscr{P}$-projective de $L$.

2.5. Contraction de $N$-complexes. La construction du $\S 2.4$ a une réciproque. Soit $(P, d)$ une $N$-résolution $\mathscr{P}$-projective d'un object $L$. Etant donné $p=1, \cdots, N-1$, nous lui associons la résolution $\mathscr{P}$-projective (traditionnelle) $\Delta_{p} P$

$$
\cdots \stackrel{d^{p}}{\longrightarrow} P_{2 N-1} \stackrel{d^{N-p}}{\longrightarrow} P_{N+p-1} \stackrel{d^{p}}{\longrightarrow} P_{N-1} \stackrel{d^{p N-p}}{\longrightarrow} P_{p-1} \stackrel{\varepsilon d^{p-1}}{\longrightarrow} L \longrightarrow 0 .
$$

Notons qu'on a des morphismes de $N$-complexes

$$
\Delta_{N-1} P \longrightarrow \Delta_{N-2} P \longrightarrow \cdots \longrightarrow \Delta_{2} P \longrightarrow \Delta_{1} P
$$


induits par la différentielle $d$ comme suit :

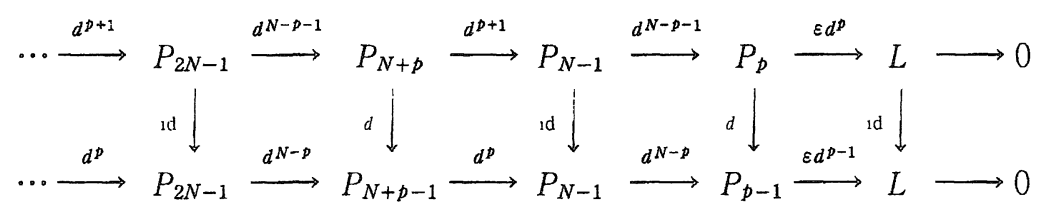

Nous énonçons maintenant l'analogue pour les $N$-complexes du lemme fondamental de l'algèbre homologique.

2.6. Lemme。 Soit $u: L \rightarrow M$ un morphisme de $\mathscr{A}$. Si $\left(P, d, \varepsilon: P_{0} \rightarrow L\right)$ est une $N$-résolution $\mathscr{P}$-projective de $L$ et $\left(Q, d, \varepsilon: Q_{0} \rightarrow M\right)$ est une $N$-résolution $\mathscr{P}$-projective de $M$, il existe un morphisme de $N$-complexes $f: P \rightarrow Q$ tel que $\varepsilon f_{0}=u \varepsilon$.

Si $g: P \rightarrow Q$ est un autre morphisme de $N$-complexes tel que $\varepsilon g_{0}=u \varepsilon$, alors $f$ et $g$ sont homotopes.

Démonstration. (a) Le $N$-complexe

$$
\cdots \stackrel{d}{\longrightarrow} Q_{1} \stackrel{d}{\longrightarrow} Q_{0} \stackrel{\varepsilon}{\longrightarrow} M \longrightarrow 0
$$

étant $\mathscr{P}$-exact, l'application $\operatorname{Hom}\left(P_{0}, Q_{0}\right) \rightarrow \operatorname{Hom}\left(P_{0}, M\right)$ est surjective. Il existe donc un morphisme $f_{0}: P_{0} \rightarrow Q_{0}$ tel que $\varepsilon f_{0}=u \varepsilon$.

Supposons construits des morphismes $f_{i}: P_{i} \rightarrow Q_{\imath}$ pour $i=0, \cdots, n$ tels que $d f_{\imath}=f_{\imath-1} d$ pour tout $1 \leq i \leq n$. Pour simplifier, posons $\varepsilon=d, f_{-1}=u$ et $f_{k}=0$ si $k<-1$. On a ainsi $d^{N}=0$ pour le $N$-complexe (2.3) tout entier et $d f_{1}=f_{1-1} d$ pour tout $i \leq n$. L'hypothèse de récurrence entraîne que

$$
d^{N-1} f_{n} d=f_{n-N+1} d^{N-1} d=f_{n-N+1} d^{N}=0 .
$$

L'exactitude de la suite

$$
\operatorname{Hom}\left(P_{n+1}, Q_{n+1}\right) \rightarrow \operatorname{Hom}\left(P_{n+1}, Q_{n}\right) \rightarrow \operatorname{Hom}\left(P_{n+1}, Q_{n-N+1}\right)
$$

implique l'existence de $f_{n+1}: P_{n+1} \rightarrow Q_{n+1}$ tel que $d f_{n+1}=f_{n} d$.

(b) Si $g: P \rightarrow Q$ est un autre morphisme de $N$-complexes tel que $\varepsilon g_{0}=u \varepsilon$, alors $\varepsilon\left(f_{0}-g_{0}\right)=u \varepsilon-u \varepsilon=0$. L'exactitude de la suite

$$
\operatorname{Hom}\left(P_{0}, Q_{N-1}\right) \rightarrow \operatorname{Hom}\left(P_{0}, Q_{0}\right) \rightarrow \operatorname{Hom}\left(P_{0}, M\right)
$$

nous donne un morphisme $h_{0}: P_{0} \rightarrow Q_{N-1}$ tel que $f_{0}-g_{0}=d^{N-1} h_{0}$.

Supposons construits des morphismes $h_{k}: P_{k} \rightarrow Q_{k+N-1}$ pour $k=0, \cdots, n$ tels que

$$
f_{k}-g_{k}=\sum_{i=0}^{N-1} d^{N-i-1} h_{k-\imath} d^{\imath}
$$

avec la convention $h_{k}=0$ si $k<0$. Alors 


$$
\begin{aligned}
d\left(f_{n+1}-g_{n+1}\right) & =\left(f_{n}-g_{n}\right) d=\sum_{i=0}^{N-1} d^{N-\imath-1} h_{n-\imath} d^{\imath+1} \\
& =\sum_{i=0}^{N-2} d^{N-\imath-1} h_{n-\imath} d^{\imath+1}=d\left(\sum_{i=1}^{N-1} d^{N-\imath-1} h_{n+1-\imath} d^{\imath}\right)
\end{aligned}
$$

Et donc $d F=0$ avec

$$
F=f_{n+1}-g_{n+1}-\left(\sum_{\imath=1}^{N-1} d^{N-\imath-1} h_{n+1-\imath} d^{i}\right) .
$$

La suite

$$
\operatorname{Hom}\left(P_{n+1}, Q_{n+N}\right) \rightarrow \operatorname{Hom}\left(P_{n+1}, Q_{n+1}\right) \rightarrow \operatorname{Hom}\left(P_{n+1}, Q_{n}\right)
$$

étant exacte, il existe un élément $h_{n+1} \in \operatorname{Hom}\left(P_{n+1}, Q_{n+N}\right)$ tel que $F=d^{N-1} h_{n+1}$. Par conséquent,

$$
\begin{aligned}
f_{n+1}-g_{n+1} & =d^{N-1} h_{n+1}+\sum_{i=1}^{N-1} d^{N-\imath-1} h_{n+1-\imath} d^{i} \\
& =\sum_{i=0}^{N-1} d^{N-\imath-1} h_{n+1-\imath} d^{i} .
\end{aligned}
$$

On construit ainsi, de proche en proche, une homotopie entre $f$ et $g$.

\section{§3. Les Groupes ${ }_{p} \operatorname{Tor}_{*}^{A}(L, M)$}

Soit $A$ une $k$-algèbre et soit ${ }_{A} \bmod \left(\operatorname{resp} . \bmod _{A}\right)$ la catégorie abélienne des $A$-modules à gauche (resp. à droite). Dans chacune de ces catégories, nous distinguons deux classes projectives, à savoir la classe projective absolue $\left(\mathscr{P}_{a}, \mathscr{\bigotimes}_{a}\right)$ et la classe projective $k$-scindée $\left(\mathscr{P}_{k}, \mathscr{\bigotimes}_{k}\right)$. Chaque classe projective fournit un bifoncteur gradué Tor ${ }_{*}^{A}(-,-)$.

Fixons l'une des classes projectives précédentes $(\mathscr{P}, \&)$ et considérons un $A$-module à droite $L$ et un $A$-module à gauche $M$.

3.1. Proposition. Soit $\left(P, d, \varepsilon: P_{0} \rightarrow L\right)$ une $N$-résolution $\mathscr{P}$-projective de $L$ ct $\left(Q, d, \varepsilon: Q_{0} \rightarrow M\right)$ une $N$-résolution $\mathscr{P}$-projective de $M$. Pour tout $p=1, \cdots, N-1$ et tout $n \in \mathbb{N}$, on $a$

$$
\begin{aligned}
{ }_{n} H_{n}\left(P \otimes_{A} M, d \otimes \mathrm{id}\right) & ={ }_{p} H_{n}\left(L \otimes_{A} Q, \mathrm{id} \otimes_{d}\right) \\
& =\left\{\begin{array}{cl}
\operatorname{Tor}_{2(n-p+1) / N}^{A}(L, M) & \text { si } n+1 \equiv p \bmod N, \\
\operatorname{Tor}_{(2 n+2-N) / N}^{A}(L, M) & \text { si } n+1 \equiv 0 \bmod N, \\
0 & \text { sinon. }
\end{array}\right.
\end{aligned}
$$


3.2. Définition. La proposition précédente montre que les groupes ${ }_{p} H_{*}(P$ $\otimes_{A} M, d \otimes$ id $)$ et ${ }_{p} H_{*}\left(L \otimes_{A} Q\right.$, id $\left.\otimes d\right)$ sont indépendants des $N$-résolutions $\mathscr{P}$-projectives choisies. Nous pouvons donc poser

$$
{ }_{p} \operatorname{Tor}_{n}^{A}(L, M)={ }_{p} H_{n}\left(P \otimes_{A} M\right)={ }_{p} H_{n}\left(L \bigotimes_{A} Q\right)
$$

où $\left(P, d, \varepsilon: P_{0} \rightarrow L\right)$ est une $N$-résolution $\mathscr{P}$-projective de $L$ et $\left(Q, d, \varepsilon: Q_{0} \rightarrow M\right)$ est une $N$-résolution $\mathscr{P}$-projective de $M$.

3.3. Corollaire. Les groupes ${ }_{p} \operatorname{Tor}_{n}^{A}(L, M)$ sont liés aux groupes $\operatorname{Tor}_{n}^{A}(L, M)$ par

$$
{ }_{p} \operatorname{Tor}_{n}^{A}(L . M)=\left\{\begin{array}{cl}
\operatorname{Tor}_{2(n-p+1) / N}^{A}(L, M) & \text { si } n+1 \equiv p \bmod N \\
\operatorname{Tor}_{(2 n+2-N) / N}^{A}(L, M) & \text { si } n+1 \equiv 0 \bmod N \\
0 & \text { sinon. }
\end{array}\right.
$$

3.4. Démonstration de la Proposition 3.1. Soit $p$ un entier compris entre 1 et $N-1$. Nous allons calculer ${ }_{p} H_{*}\left(P \otimes_{A} M, d \otimes \mathrm{id}\right)$. Les groupes ${ }_{p} H_{*}\left(L \bigotimes_{A} Q, \mathrm{id} \bigotimes_{d}\right)$ se calculent par la même méthode.

Le complexe $\Delta_{p} P$ construit au $\S 2.5$ à partir de la $N$-résolution $\mathscr{P}$-projective est une résolution $\mathscr{P}$-projective de $L$ au sens usuel. Il en résulte que Tor* $(L, M)$ est l'homologie du complexe $\Delta_{p} P \otimes_{A} M=\Delta_{p}\left(P \otimes_{A} M\right)$ :

$$
\cdots \stackrel{\left(d \otimes_{1 \mathrm{~d}}\right)^{p}}{\longrightarrow} P_{2 N-1} \otimes_{A} M \stackrel{\left(d \otimes_{1 \mathrm{~d}}\right)^{N-p}}{\longrightarrow} P_{N+p-1} \otimes_{A} M \stackrel{\left(d \otimes_{1 \mathrm{~d}}\right)^{p}}{\longrightarrow} P_{N-1} \otimes_{A} M \stackrel{\left(d \otimes_{1 \mathrm{~d}}\right)^{N-p}}{\longrightarrow} P_{p-1} \otimes_{A} M \longrightarrow 0 .
$$

Par conséquent.

$$
\operatorname{Tor}_{2 n}^{A}(L, M)={ }_{p} H_{n N+p-1}\left(P \otimes_{A} M\right) \quad \text { et } \operatorname{Tor}_{2 n+1}^{A}(L, M)={ }_{N-p} H_{(n+1) N-1}\left(P \bigotimes_{A} M\right)
$$

pour tout entier $n \geq 0$. En particulier, le groupe ${ }_{N-p} H_{(n+1) N-1}\left(P \bigotimes_{A} M\right)$ ne dépend pas de $p$, ce qui nous permet d'écrire

$$
\operatorname{Tor}_{2 n+1}^{A}(L, M)={ }_{p} H_{(n+1) N-1}\left(P \otimes_{A} M\right) .
$$

On en déduit aussitôt le calcul de ${ }_{p} H_{*}\left(P \otimes_{A} M, d \otimes\right.$ id $)$ lorsque $n+1 \equiv 0$ ou $p$ modulo $N$. Si $N=2$ il n'y a plus rien à démontrer.

Si $N>2$, il nous reste à établir la nullité de ${ }_{p} H_{n}\left(P \otimes_{A} M, d \otimes\right.$ id $)$ lorsque $n+1$ $\not \equiv 0, p$ modulo $N$. Pour alléger les notations, posons ${ }_{p} H_{n}\left(P \otimes_{A} M, d \otimes \mathrm{id}\right)={ }_{p} H_{n}$.

Du morphisme de résolutions $\Delta_{p+1} P \rightarrow \Delta_{p} P$ défini au $\S 2.5$, nous déduisons un quasi-isomorphisme $\Delta_{p+1} P \bigotimes_{A} M \rightarrow \Delta_{p} P \bigotimes_{A} M$. En particulier, en degré pair $2 k$, il induit l'isomorphisme

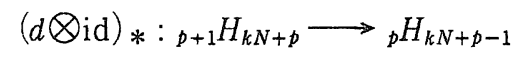

qui n'est autre que le morphisme $d_{*}$ du $§ 1.5$. En degré impair $2 k-1$, il induit 
l'isomorphisme

$$
\mathrm{id} *:{ }_{N-p-1} H_{k N-1} \longrightarrow{ }_{N-p} H_{k N-1}
$$

qui s'identifie avec le morphisme $i_{*}$ du $\S 1.5$.

Soit $r>0$ un entier tel que $p+r<N$. Du lemme 1.6 nous tirons la suite exacte

$$
\begin{aligned}
& { }_{r} \mathrm{H}_{k N-1} \stackrel{{ }^{\iota_{*}^{*}} \longrightarrow}{\longrightarrow}{ }_{r+p} \mathrm{H}_{k N-1} \stackrel{d_{+}^{\prime}}{\longrightarrow}{ }_{p} H_{k N-1-r} \\
& { }_{2 \rightarrow+\infty}^{N \rightarrow-1} \\
& { }_{N-r} H_{k N-1-r} \stackrel{d_{+}^{p}}{\longrightarrow}{ }_{N-r-p} H_{k N-1-r-p} .
\end{aligned}
$$

D'après ce qui précède, le premier morphisme $i_{*}^{p}$ est un isomorphisme; il en est de même du dernier morphisme $d_{*}^{p}$. On en déduit que le groupe ${ }_{p} H_{k N-1-r}$ est nul. En d'autres termes, ${ }_{p} H_{n}=0$ lorsque $n+1 \equiv N-r$ modulo $N$. Comme les conditions $r>0$ et $r+p<N$ sont équivalentes à la condition $p<N-r<N$, on a démontré que ${ }_{p} H_{n}=0$ lorsque $n+1 \equiv s$ avec $p<s<N$.

Pour établir que ${ }_{p} H_{n}=0$ lorsque $n+1 \equiv s$ avec $0<s<p$, prenons un entier $r$ tel que $0<_{r}<p$ et $r+p<N$, et considérons la suite exacte également tirée du lemme 1.6

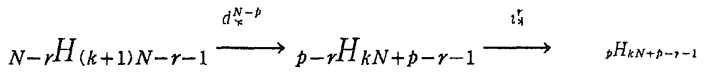

$$
\begin{aligned}
& d a^{-+} \downarrow \\
& { }_{r} H_{k N-1} \stackrel{i_{x}^{N-p}}{\longrightarrow}{ }_{N-p+r} H_{k N-1} .
\end{aligned}
$$

Comme précédemment, les morphismes extrêmes sont des isomorphismes. On en déduit que ${ }_{p} H_{k N+p-r-1}=0$. Ainsi, on a prouvé que ${ }_{p} H_{n}=0$ lorsque $n+1 \equiv p-r$ modulo $N$. On conclut en remarquart que $p-r$ parcourt tous les entiers $s$ tels que $0<s<p$.

3.5. Fonctorialité. Les modules ${ }_{p} \operatorname{Tor}_{n}^{A}(L, M)$ sont fonctoriels en $L$ et $M$. Cela résulte du lemme 2.6 ou de l'identification donnée par le corollaire 3.3.

3.6. Proposition. (a) Soit $0 \rightarrow M^{\prime} \rightarrow M \rightarrow M^{\prime \prime} \rightarrow 0$ une suite $\mathscr{P}$-exacte courte de $N$-complexes de $A$-modules à gauche. Pour tout $A$-module à droite $L$ il existe une longue suite exacte naturelle

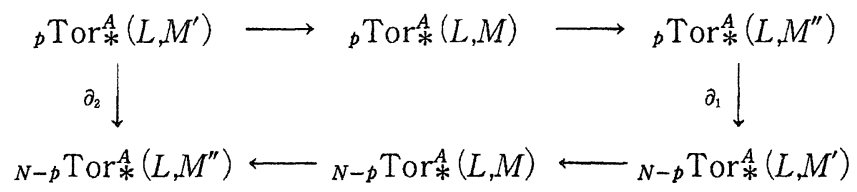


(b) De même, si $0 \rightarrow L^{\prime} \rightarrow L \rightarrow L^{\prime \prime} \rightarrow 0$ est une suite $\not 9$-exacte courte de $N$-complexes de $A$-modules à droite et si $M$ est un $A$-module à gauche, alors il existe une longue suite exacte naturelle

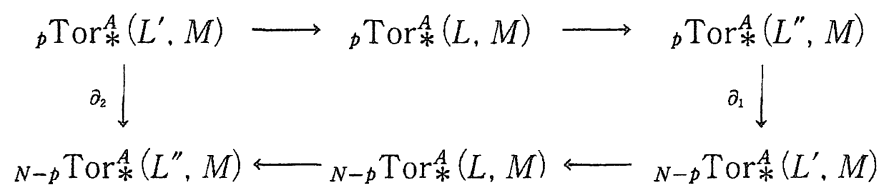

Dans les cas $(a)$ et $(b)$. l'application $\partial_{1}$ est de degré $-p$ et $\partial_{2}$ de degré $-(N-p)$.

Démonstration. Nous nous contenterons d'établir (a). Le point (b) se démontre de manière similaire.

Soit $\left(P, d, \varepsilon: P_{0} \rightarrow L\right)$ une $N$-résolution $\mathscr{P}$-projective de $L$. Considérons la suite de $N$-complexes

$$
0 \rightarrow P \otimes_{A} M^{\prime} \rightarrow P \otimes_{A} M \rightarrow P \otimes_{A} M^{\prime \prime} \rightarrow 0 .
$$

La suite (3.2) est exacte à droite. Si nous montrons que $P_{n} \otimes_{A} M^{\prime} \rightarrow P_{n} \otimes_{A} M$ est injectif pour tout $n \geq 0$. alors la suite (3.2) est exacte, ce qui permet de lui appliquer le lemme 1.8 et de conclure.

Lorsque nous sommes dans le cas de la classe projective absolue, le $N$-complexe est constitué de $A$-modules qui sont facteurs directs de modules libres. L'injectivité de $P_{n} \otimes_{A} M^{\prime} \rightarrow P_{n} \otimes_{A} M$ se ramène au cas où $P_{n}$ est un $A$-module libre, c'est-à-dire un cas où l'injectivité est triviale.

Lorsque nous sommes dans la situation de la classe projective $k$-scindée $\left(\mathscr{P}_{k}, \bigotimes_{k}\right)$, alors $P_{n}$ est de la forme $V \bigotimes_{k} A$ avec la structure naturelle de $A$-module à droite. L'exactitude de (3.2) se rédıit alors à celle de la suite de $k$-modules

$$
0 \rightarrow V \bigotimes_{k} M^{\prime} \rightarrow V \bigotimes_{k} M \rightarrow V \bigotimes_{k} M^{\prime \prime} \rightarrow 0
$$

pour tout $k$-module $V$. Cette dernière est bien exacte. En effet, la $\mathscr{P}_{k}$-exactitude de la suite $0 \rightarrow M^{\prime} \rightarrow M \rightarrow M^{\prime \prime} \rightarrow 0$ implique qu'elle est exacte et scindée dans la catégorie des $k$-modules (et réciproquement), propriété qui est préservée par le foncteur $V \bigotimes_{k}-$.

3.7. Caractérisation des bifoncteurs $p$ Tor. La famille de bifoncteurs gradués ${ }_{p} \operatorname{Tor}_{*}^{A}(-,-)(0<p<N)$ est caractérisée par les trois propriétés (1), (11), (111) ci-dessous où $L$ désigne un $A$-module à droite et $M$ un $A$-module à gauche.

(i) Pour tout $p$ tel que $0<p<N$, on a

$$
{ }_{p} \operatorname{Tor}_{p-1}^{A}(L, M) \cong L \bigotimes_{A} M .
$$

(ii) Si $P$ et $Q$ sont des modules $\mathscr{P}$-projectifs et si $n \neq p-1$, on a 


$$
{ }_{p} \operatorname{Tor}_{n}^{A}(P, M)=0={ }_{p} \operatorname{Tor}_{n}^{A}(L, Q) .
$$

(iii) La proposition 3.6 est vérifiée.

Pour la démonstration, on procède comme dans le cas classique.

\section{§4. Les Groupes $\operatorname{Ext}_{A}^{*}(L, M)$}

Soit $A$ une $k$-algèbre et ${ }_{A} \bmod$ la catégorie abélienne des $A$-modules à gauche. Nous nous restreignons volontairement aux deux classes projectives $\left(\mathscr{P}_{a}, \mathscr{S}_{a}\right)$ et $\left(\mathscr{P}_{k}, \mathscr{\bigotimes}_{k}\right)$ considérées aux $\S \S 2-3$. A chacune de ces classes correspond la classe $\mathscr{I}$ formée de tous les $A$-modules $I$ tels que la suite

$$
\operatorname{Hom}_{A}\left(Y^{\prime \prime}, I\right) \rightarrow \operatorname{Hom}_{A}(Y, I) \rightarrow \operatorname{Hom}_{A}\left(Y^{\prime}, I\right)
$$

soit exacte pour toute suite $Y^{\prime} \stackrel{u}{\rightarrow} Y \stackrel{v}{\rightarrow} Y^{\prime \prime}$ de $\mathscr{S}$. Un élément de $\mathscr{I}$ est appelé un module $\mathscr{I}$-injectif.

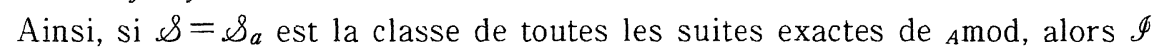
est constituée de ce que l'on appelle d'habitude les $A$-modules injectifs. Par contre, si $\mathscr{S}=\bigotimes_{k}$. alors $\mathscr{I}$ est la classe des $A$-modules de la forme $\operatorname{Hom}_{k}(A, V)$ sur lesquels $A$ opère par $(a f)\left(a^{\prime}\right)=f\left(a^{\prime} a\right)$ pour tout $f \in \operatorname{Hom}_{k}(A . V)$ et $a . a^{\prime} \in A$.

4.1. Définition. Un $N$-complexe $(C, d)$ est $\mathscr{I}$-exact si le $N$-complexe $\operatorname{Hom}_{A}(C . I)$ est acyclique pour tout objet $\mathscr{I}$-injectif $I$.

Une $N$-résolution $\mathscr{I}$-injective d'un $A$-module $L$ est la donnée d'un $N$-complexe $\mathscr{I}$-exact

$$
0 \longrightarrow L \stackrel{n}{\longrightarrow} I_{0} \stackrel{d}{\longrightarrow} I_{-1} \stackrel{d}{\longrightarrow} I_{-2} \stackrel{d}{\longrightarrow} \cdots
$$

tel que le module $I_{n}$ est $\mathscr{I}$-injectif pour tout $n \leq 0$.

Nous noterons $\left(I, d, \eta: L \rightarrow I_{0}\right)$ la donnée d'une $N$-résolution $\mathscr{I}$-injective de $L$.

\subsection{Rapport avec les résolutions injectives traditionnelles.} L'existence de $N$-résolutions $\mathscr{I}$-injectives se démontre comme au $\$ 2.4$. On part d'une résolution $\mathscr{I}$-injective de $L$ au sens de l'algèbre homologique usuelle

$$
0 \longrightarrow L \stackrel{\eta}{\longrightarrow} J_{0} \stackrel{d}{\longrightarrow} J_{-1} \stackrel{d}{\longrightarrow} J_{-2} \stackrel{d}{\longrightarrow} \cdots
$$

On obtient une $N$-résolution $\mathscr{I}$-injective de $L$ en insérant l'application identité $N-2$ fois à la place de tous les modules $J_{-21}(i \geq 0)$.

Réciproquement, si on part de la $N$-résolution $\mathscr{I}$-injective (4.1), on a, pour tout $p$ tel que $0<p<N$, la résolution $\mathscr{I}$-injective usuelle $\Delta^{p} J$

$$
0 \rightarrow L \stackrel{d^{p-1} \eta}{\longrightarrow} I_{-(p-1)} \stackrel{d^{N-p}}{\longrightarrow} I_{-(N-1)} \stackrel{d^{p}}{\longrightarrow} I_{-(N+P-1)} \stackrel{d^{N-p}}{\longrightarrow} I_{-(2 N-1)} \stackrel{d^{p}}{\longrightarrow} \cdots
$$


Les résolutions $\Delta^{p} I$ sont liées par des morphismes de $N$-complexes

$$
\Delta^{1} P \rightarrow \Delta^{2} P \rightarrow \cdots \rightarrow \Delta^{N-2} P \rightarrow \Delta^{N-1} P
$$

définis comme suit:

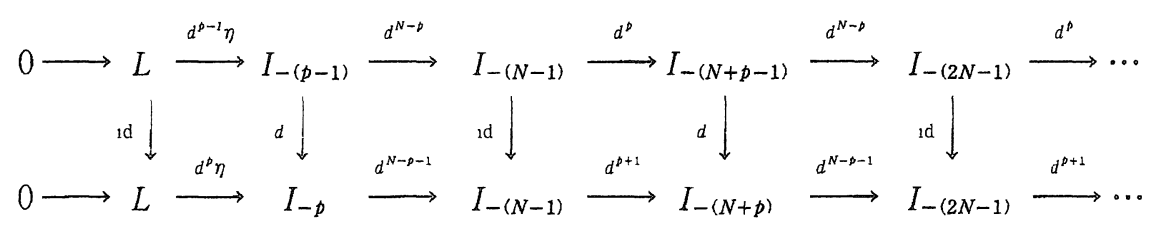

Le lemme 2.6 a le pendant suivant dont nous laissons la démonstration au lecteur.

4.3. Lemme. Soit $u: L \rightarrow M$ un morphisme de ${ }_{A} \bmod$. Si $\left(I, d, \eta: L \rightarrow I_{0}\right)$ est une $N$-résolution $\mathscr{I}$-injective de $L$ et $\left(J, d, \eta: M \rightarrow J_{0}\right)$ est une $N$-résolution $\mathscr{I}$-injective de $M$, il existe un morphisme de $N$-complexes $f: I \rightarrow J$ tel que $f_{0} \eta=\eta u$.

Si $g: I \rightarrow J$ est un autre morphisme de $N$-complexes tel que $g_{0} \eta=\eta u$, alors $f$ et $g$ sont homotopes.

Nous fixons maintenant l'une des deux classes projectives $\left(\mathscr{P}_{a}, \mathscr{S}_{a}\right)$ et $\left(\mathscr{P}_{k}, \mathscr{S}_{k}\right)$ de la catégorie abélinne ${ }_{A}$ mod. Il lui correspond une classe injective $\mathscr{I}$ et des bifoncteurs Ext娄 $(-,-)$.

Considérons des $A$-modules à gauche $L$ et $M$. Soit $\left(P, d, \varepsilon: P_{0} \rightarrow L\right)$ une $N$-résolution $\mathscr{P}$-projective de $L$ et $\left(I, d, \eta: M \rightarrow I_{0}\right)$ une $N$-résolution $\mathscr{I}$-injective de $M$. Graduons $\operatorname{Hom}_{A}(L, I)$ et $\operatorname{Hom}_{A}(P, M)$ par

$$
\operatorname{Hom}_{A}(L, I)_{n}=\operatorname{Hom}_{A}\left(L, I_{n}\right) \text { et } \operatorname{Hom}_{A}(P, M)_{n}=\operatorname{Hom}_{A}(P-n, M)
$$

et munissons-les des endomorphismes de degré -1 donnés respectivement par $f$ $\mapsto d \circ f$ et $g \mapsto g \circ d$ pour tout $f \in \operatorname{Hom}_{A}(L, I)$ et $g \in \operatorname{Hom}_{A}(P, M)$. On obtient ainsi des $N$-complexes négatifs.

4.4. Proposition. Sous les hypothèses précédentes, pour tout $p=1, \cdots, N-1$ et tout $n \in \mathbb{N}$, on a

$$
\begin{aligned}
{ }_{p} H_{-n}\left(\operatorname{Hom}_{A}(L, I)\right) & ={ }_{p} H_{-n}\left(\operatorname{Hom}_{A}(P, M)\right) \\
& =\left\{\begin{array}{cl}
\operatorname{Ext}_{A}^{2(n+1-(N-p) / N}(L, M) & \text { si } n+1 \equiv N-p \bmod N, \\
\operatorname{Ext}_{A}^{(2 n+2-N) / N}(L, M) & \text { si } n+1 \equiv 0 \bmod N, \\
0 & \text { sinon. }
\end{array}\right.
\end{aligned}
$$

Démonstration. Le complexe $\Delta_{p} P$ construit au $\S 2.5$ est une résolution $\mathscr{P}$-projective de $L$ au sens usuel. De même, $\Delta^{p} I$ est une résolution $\mathscr{I}$-injective de $M$. On peut donc calculer les groupes $\operatorname{Ext}_{A}^{*}(L, M)$ à partir des complexes $\operatorname{Hom}_{A}\left(L, \Delta^{p} I\right)$ et $\operatorname{Hom}_{A}\left(\Delta_{p} P, M\right)$. On obtient pour tout $n \in \mathbb{N}$ 


$$
\begin{aligned}
\operatorname{Ext}_{A}^{2 n}(L, M) & ={ }_{N-p} H_{-(n N+p-1)}\left(\operatorname{Hom}_{A}(L, I)\right)={ }_{p} H_{-(n N+N-p-1)}\left(\operatorname{Hom}_{A}(L, I)\right) \\
& ={ }_{N-p} H_{-(n N+p-1)}\left(\operatorname{Hom}_{A}(P, M)\right)={ }_{p} H_{-(n N+N-p-1)}\left(\operatorname{Hom}_{A}(P, M)\right)
\end{aligned}
$$

et

$$
\operatorname{Ext}_{A}^{2 n+1}(L, M)={ }_{p} H_{-[(n+1) N-1]}\left(\operatorname{Hom}_{A}(L, I)\right)={ }_{p} H_{-[(n+1) N-1]}\left(\operatorname{Hom}_{A}(P, M)\right) .
$$

On en déduit, comme au $§ 3.4$, le calcul de ${ }_{p} H_{-n}\left(\operatorname{Hom}_{A}(L, I)\right)$ et de ${ }_{p} H_{-n}\left(\operatorname{Hom}_{A}(P, M)\right)$ lorsque $n+1 \equiv 0$ ou $N-p$ modulo $N$.

Quant aux autres cas, on les traite au moyen des morphismes de résolutions (4.3) en procédant comme au $\$ 3.4$.

4.5. Définition. La proposition précédente montre que les groupes ${ }_{p} H_{*}$ $\left(\operatorname{Hom}_{A}(L, I)\right)$ et ${ }_{p} H_{*}\left(\operatorname{Hom}_{A}(P, M)\right)$ sont indépendants des $N$-résolutions choisies. Nous pouvons donc poser

$$
{ }_{p} \operatorname{Ext}_{A}^{n}(L, M)={ }_{p} H_{-n}\left(\operatorname{Hom}_{A}(L, I)\right)={ }_{p} H_{-n}\left(\operatorname{Hom}_{A}(P, M)\right)
$$

où $\left(P, d, \varepsilon: P_{0} \rightarrow L\right)$ est une $N$-résolution $\mathscr{P}$-projective de $L$ et $\left(I, d, \eta: M \rightarrow I_{0}\right)$ est une $N$-résolution $\mathscr{I}$-injective de $M$.

4.6. Corollaire. Les groupes ${ }_{p} \operatorname{Ext}_{A}^{n}(L, M)$ sont donnés par

$$
{ }_{p} \operatorname{Ext}_{A}^{n}(L, M)=\left\{\begin{array}{cl}
\operatorname{Ext}_{A}^{2(n+1-(N-p)) / N}(L, M) & \text { si } n+1 \equiv N-p \bmod N, \\
\operatorname{Ext}_{A}^{(2 n+2-N) / N}(L, M) & \text { si } n+1 \equiv 0 \bmod N, \\
0 & \text { sinon. }
\end{array}\right.
$$

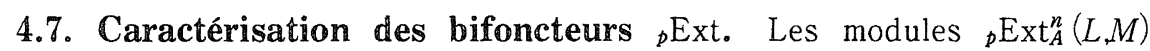
sont fonctoriels en les $A$-modules à gauche $L$ et $M$. Ils sont caractérisés par les propriétiés (i ) - (iv) ci-dessous.

(i) Pour tout $p$ tel que $0<p<N$, on a

$$
{ }_{p} \operatorname{Ext}_{A}^{N-p-1}(L, M) \cong \operatorname{Hom}_{A}(L, M) .
$$

(ii) Pour tous $n$ et $p$ tels que $n \neq N-p-1$, tout module $\mathscr{P}$-projectif $P$ et tout module $\mathscr{I}$-injectif $I$, on a

$$
{ }_{p} \operatorname{Ext}_{A}^{n}(P, M)=0={ }_{p} \operatorname{Ext}_{A}^{n}(L, I) .
$$

(iii) Soit $0 \rightarrow M^{\prime} \rightarrow M \rightarrow M^{\prime \prime} \rightarrow 0$ une suite $\mathscr{P}$-exacte courte de $N$-complexes de $A$-modules. Pour tout $A$-module $L$ il existe une longue suite exacte naturelle 


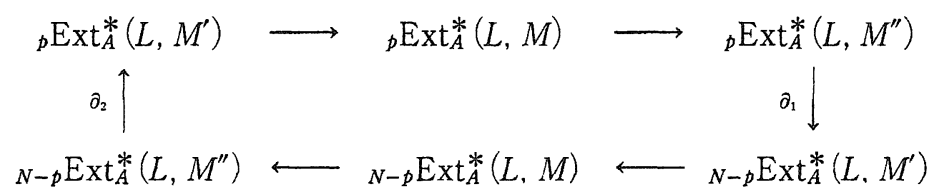

(iv) De même, si $0 \rightarrow L^{\prime} \rightarrow L \rightarrow L^{\prime \prime} \rightarrow 0$ est une suite $\mathscr{I}$-exacte courte de $N$-complexes de $A$-modules et si $M$ est un $A$-module quelconque, alors il existe une longue suite exacte naturelle

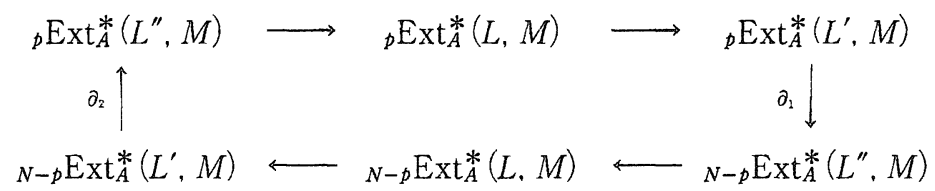

Les applications $\partial_{1}$ et $\partial_{2}$ ci-dessus sont de degrés respectifs $p$ et $N-p$.

Dans la démonstration de l'exactitude des longues suites précédentes pour le cas où $\&=\oiint_{k}$, on utilise les isomorphismes d'adjonction

$$
\operatorname{Hom}_{A}\left(A \otimes_{k} V, L\right) \cong \operatorname{Hom}_{k}(V, L) \text { et } \operatorname{Hom}_{A}\left(L, \operatorname{Hom}_{k}(A, V)\right) \cong \operatorname{Hom}_{k}(L, V)
$$

où $L$ est un $A$-module à gauche et $V$ un $k$-module.

\section{§5. Démonstration du Théorème $\mathbb{1}$}

Dans ce paragraphe nous aurons besoin des deux hypothèses $\left(\mathrm{H}_{0}\right)$ et $\left(\mathrm{H}_{1}\right)$ suivantes, déjà mentionnées dans l'introduction. Elles portent sur l'entier $N \geq 2$. l'anneau commutatif $k$ et l'élément $q$ de $k$.

$\left(\mathrm{H}_{0}\right)$ On a $[N]=0$ dans $k$.

$\left(\mathrm{H}_{1}\right)$ L'hypothèse $\left(\mathrm{H}_{0}\right)$ est vérifiée et, de plus, $[p]$ est inversible dans $k$ pour tout $0<p<N$.

L'hypothèse $\left(\mathrm{H}_{0}\right)$ est équivalente à : soit $q$ est une racine $N$-ième de l'unité différente de 1 , soit $q=1$ et $N=0$ dans $k$.

L'hypothèse $\left(\mathrm{H}_{1}\right)$ est équivalente á: soit $q$ est une racine primitive $N$-ième de l'unité, soit $q=1$, l'entier $N$ est premier et $k$ est une algèbre sur le corps $\mathbb{Z} / N$.

5.1. Modules simpliciaux. Soit $\left(C, d_{2}, s_{l}\right)$ un $k$-module simplicial muni d'applications face $d_{\imath}$ et dégénérescence $s_{\imath}$. On a notamment les formules de commutation simpliciales

$$
d_{i} d_{\jmath}=d_{j-1} d_{\imath} \text { si } i<j \text { et } d_{\imath} s_{\jmath}= \begin{cases}s_{\jmath-1} d_{\imath} & \text { si } i<j, \\ \text { id } & \text { si } i=j, j+1, \\ s_{\jmath} d_{\imath-1} & \text { si } i>j+1 .\end{cases}
$$

Pour tout scalaire $q \in k$, on définit $d: C_{n} \rightarrow C_{n-1}$ par 


$$
d=\sum_{i=0}^{n} q^{\imath} d_{i}
$$

Kapranov ([5], Proposition 0.2) et Dubois-Violette ([1], Lemme 3) ont observé que l'on a $d^{N}=0$ sous l'hypothèse $\left(\mathrm{H}_{0}\right)$.

5.2. Proposition. Avec les notations précédentes, définissons $\delta: C_{n} \rightarrow C_{n-1}$ par

$$
\delta=\sum_{i=0}^{n-1} q^{\imath} d_{1}
$$

On a $\delta^{N}=0$ sous l'hypothèse $\left(\mathrm{H}_{0}\right)$; si, de plus, l'hypothèse $\left(\mathrm{H}_{1}\right)$ est vérifiée, alors $(C, \delta)$ est $N$-complexe acyclique.

Nous allons nous placer dans la situation plus générale suivante. Supposons que $\delta: C_{n} \rightarrow C_{n-1}$ soit de la forme

$$
\delta=\sum_{i=0}^{n-1} a_{n-1-\imath} q^{\imath} d_{i}
$$

où $a_{0}, a_{1}, \cdots$ sont des scalaires fixés une fois pour toutes.

5.3. Lemme. Si l'on pose $(N, j, k)=\sum_{s=0}^{N-k} q^{s} a_{n-k-\jmath-s}$, on a

$$
\delta^{N}=\sum_{0 \leq i_{1} \leq i_{i} \leq \cdots \leq i_{N} \leq n-N} q^{i_{1}+12+\cdots+i_{N}} \prod_{k=1}^{N}\left(N, i_{k}, k\right) d_{l_{N}} d_{i_{N-1}} \cdots d_{{ }_{12}} d_{i_{1}} .
$$

Démonstration. Nous allons procéder par récurrence sur l'entier $N$. La formule est vérifiée lorsque $N=1$. Supposons qu'elle le soit pour un entier $N \geq 1$. Nous avons

$$
\begin{aligned}
& \delta^{N+1}=\delta \delta^{N}=\left(\sum_{j=0}^{n-N-1} a_{n-N-1-\jmath} q^{\jmath} d_{\jmath}\right) \delta^{N} \\
& =\sum_{0 \leq 1_{1} \leq \cdots \leq i_{v} \leq n-N} q^{\imath^{1+\cdots+i_{N}+j}} a_{n-N-1-} \prod_{k=1}^{N}\left(N, i_{k}, k\right) d_{j} d_{i_{N}} \cdots \\
& d_{i_{1}} \\
& 0 \leq \leq_{n}-N-1 \\
& =\sum_{r=0}^{N} S_{r}
\end{aligned}
$$

où $S_{r}$ est la somme des termes précédents pour lesquels on a l'encadrement $i_{r} \leq j$ $<i_{r+1}$ si $0 \leq r \leq N-1$ (convenons que $i_{0}=0$ ) et $i_{N} \leq_{j}$ si $r=N$. Des relations de commutation simpliciales on tire pour $r<N$

$$
S_{r}=\sum q^{\imath_{1}+\cdots+\imath_{N+j}} a_{n-N-1-\jmath} \prod_{k=1}^{N}\left(N, i_{k}, k\right) d_{\imath_{N-1}} \cdots d_{r_{r+1-1}} d_{\jmath} d_{\imath r} \cdots d_{\imath 1} .
$$


En renommant les indices, on obtient

$$
S_{r}=\sum q^{i_{1}+\cdots+i_{N+1}} q^{N-r} a_{n-N-1-i_{r+1}} \prod_{k=1}^{r}\left(N, i_{k}, k\right) \prod_{k=r+1}^{N}\left(N, i_{k+1}+1, k\right) d_{i_{N+1}} d_{i_{N}} \cdots d_{\imath_{1}}
$$

où la somme est prise sur $0 \leq i_{1} \leq \cdots \leq i_{N+1} \leq n-N-1$. Posons

$$
\Phi_{r}=q^{N-r} a_{n-N-1-i r+1} \prod_{k=1}^{r}\left(N, i_{k}, k\right) \prod_{k=r+1}^{N}\left(N, i_{k+1}+1, k\right)
$$

si $0 \leq r \leq N-1$; sinon,

$$
\Phi_{N}=a_{n-N-1-i_{N+1}} \prod_{k=1}^{N}\left(N, i_{k}, k\right) .
$$

En sommant $\operatorname{sur} r=0, \cdots, N$, on a

$$
\delta^{N+1}=\sum_{0 \leq i_{1} \leq \cdots \leq i_{N+1} \leq n-N-1} q^{i_{1}+\cdots+i_{N+1}}\left(\sum_{r=0}^{N} \Phi_{r}\right) d_{i_{N+1}} \cdots d_{\imath_{1}} .
$$

Il ne reste plus qu'à montrer que

$$
\sum_{r=0}^{N} \Phi_{r}=\prod_{k=1}^{N+1}\left(N+1, i_{k}, k\right) .
$$

Remarquons que, pour tous les entiers $i, k$,

$$
(N+1, i, k)-(N . i, k)=q^{N+1-k} a_{n-1-N-\imath} \text { et }(N . i+1, k)=(N+1, i, k+1) .
$$

Par conséquent, lorsque $r<N$, on a

$$
\begin{aligned}
\Phi_{r} & =\left[\left(N+1, i_{r+1}, r+1\right)-\left(N, i_{r+1}, r+1\right)\right] \prod_{k=1}^{r}\left(N, i_{k}, k\right) \prod_{k=r+1}^{N}\left(N+1, i_{k+1}, k+1\right) \\
& =\prod_{k=1}^{r}\left(N, i_{k}, k\right) \prod_{k=r+1}^{N+1}\left(N+1, i_{k}, k\right)-\prod_{k=1}^{r+1}\left(N, i_{k}, k\right) \prod_{k=r+2}^{N+1}\left(N+1, i_{k}, k\right)
\end{aligned}
$$

et $\Phi_{N}=\prod_{k=1}^{N+1}\left(N, i_{k}, k\right)$. Les termes de la somme $\sum_{r=0}^{N} \Phi_{r}$ s'élimient deux à deux sauf $\prod_{k=1}^{N+1}\left(N+1, i_{k}, k\right)$.

๖.4. Corolliaire. Définissons $\delta: C_{n} \rightarrow C_{n-1}$ par

$$
\delta=\sum_{i=0}^{n-2} q^{i} d_{i}+[\ell] q^{n-1} d_{n-1}
$$

où $\ell$ est un entier quelconque. Alors $\delta^{N}=0$ sous l'hypothèse $\left(\mathrm{H}_{0}\right)$.

Démonstration. Comme $[\ell]=[m]$ lorsque $\ell \equiv m$ modulo $N$, nous pouvons nous limiter au cas où $0 \leq \ell \leq N-1$.

Supposons dans un premier temps que $\ell=1$. Nous appliquons le lemme 5.3 
au cas où $a_{t}=1$ pour tout $i \geq 0$. Le produit $\prod_{k=1}^{N}\left(N, i_{k}, k\right)$ est nul, et donc $\delta^{N}=0$. car

$$
\left(N, i_{1}, 1\right)=\sum_{s=0}^{N-1} q^{s} a_{n-1-i_{1}-s}=\sum_{s=0}^{N-1} q^{s}=[N]=0 .
$$

Si maintenant $\ell=0$, alors $\delta=\sum_{t=0}^{n-2} q^{t} d_{i}$. En remplaçant $n$ par $n-1$, on se ramène au cas $\ell=1$.

Si $\ell>1$ on applique le lemme 5.3 avec $a_{0}=[\ell]$ et $a_{i}=1$ pour tout $i \geq 1$. Montrons à nouveau que le produit $\prod_{k=1}^{N}\left(N, i_{k}, k\right)$ est nul en établissant la nullité d'un de ses facteurs.

Lorsque $i_{1}<n-N$, on a $a_{n-N-\imath_{1}+r}=1$ pour $r \geq 0$. Alors le facteur $(N, i, 1)$ est nul en vertu du même calcul que dans le cas $\ell=1$.

Lorsque $i_{1}=n-N$, il en est a fortiori de même de $i_{\ell}$. Maintenant, c'est le facteur $\left(N, i_{\ell}, \ell\right)$ qui est nul. En effet,

$$
\begin{aligned}
\left(N, i_{\ell, \ell} \ell\right) & =\sum_{s=0}^{N-\ell} q^{s} a_{n-\ell-1 \ell-s}=\sum_{s=0}^{\Lambda-\ell} q^{s} a_{\Lambda-\ell-s} \\
& =q^{N-\ell}[\ell]+\sum_{s=0}^{N-\ell-1} q^{s}=q^{N-\ell}[\ell]+[N-\ell] \\
& =q^{N-\ell}[\ell]+[-\ell]=0 .
\end{aligned}
$$

En conclusion, $\delta^{N}=0$.

Avant de passer à la démonstration de la Proposition 5.2. démontrons deux relations dans la $k$-algèbre $\mathscr{D}_{q}$ engendrée par les variables $X$ et $Y$ et la relation $Y X-q X Y=1$.

5.5. Lemme. Sous l'hypothèse $\left(\mathrm{H}_{1}\right)$ on a les relations suivantes dans $\mathscr{D}_{q}$ :

$$
\begin{gathered}
\sum_{k=0}^{N-1} X^{N-k-1} Y^{N-1} X^{k}=[N-1] ! \\
\sum_{k=0}^{N-1} Y^{N-k-1} X^{N-1} Y^{k}=(-1)^{N-1} q^{-N(N-1) / 2}[N-1] !
\end{gathered}
$$

Démonstration. Commençons par établir la première relation. Remarquons tout d'abord que dans $\mathscr{D}_{q}$ on a la formule

\footnotetext{
1 L'algèbre $\mathscr{D}_{q}$ s'obtient aussi comme extension d'Ore de l'algèbre de polynômes $k[X]$ à l'aide des opérateurs $\partial_{q}$ et $\tau_{q}$ définis plus bas. La formule (5.4) est un cas particulier d'identités du même type valides dans toute extension d'Ore.
} 


$$
Y^{\ell} X^{k}=\sum_{r=0}^{\ell} q^{(\ell-r)(k-r)}(\ell-r, r)[k][k-1] \cdots[k-r+1] X^{k-r} Y^{\ell-r}
$$

pour tout $k, \ell \geq 0$. Elle peut se démontrer par récurrence sur $\ell$. Pour $\ell=0$, il n'y a rien à établir. Pour $\ell=1$ la formule (5.4) a la forme

$$
Y X^{k}=q^{k} X^{k} Y+[k] X^{k-1}
$$

formule qu'on établit par récurrence sur $k$. Pour $\ell>1$, on calcule $Y^{\ell} X^{k}=$ $Y^{Y}\left(Y^{\ell-1} X^{k}\right)$ à partir de la formule (5.4) pour $Y^{\ell-1} X^{k}$ et de (5.5).

En utilisant (5.4) on obtient

$$
\begin{aligned}
& \sum_{k=0}^{N-1} X^{N-k-1} Y^{N-1} X^{k} \\
& \quad=\sum_{k=0}^{N-1} \sum_{r=0}^{N-1} q^{(N-1-r)(k-r)}(N-1-r, r)[k][k-1] \cdots[k-r+1] X^{N-1-r} Y^{N-1-r} \\
& =\sum_{r=0}^{N-1}(N-1-r, r) a(r) X^{N-1-r} Y^{N-1-r}
\end{aligned}
$$

où

$$
a(r)=\sum_{k=r}^{N-1} q^{(N-1-r)(k-r)}[k][k-1] \cdots[k-r+1]
$$

Nous affirmons que $a(r)=0$ pour $0 \leq r<N-1$. Il en résulte que

$$
\sum_{k=0}^{N-1} X^{N-k-1} Y^{N-1} X^{k}=(0, N-1) a(N-1)=[N-1] ! .
$$

ce qui démontre la première identité du lemme 5.5.

Pour établir la nullité de $a(r)$ lorsque $0 \leq r<N-1$, introduisons sur l'algèbre de polynômes $k[X]$ l'opérateur aux $q$-différences $\partial_{q}$ défini par

$$
\partial_{q}\left(X^{k}\right)=[k] X^{k-1}
$$

pour tout $k \geq 0$. Alors

$$
\begin{aligned}
a(r) & =\left.\sum_{k=r}^{N-1}[k][k-1] \cdots[k-r+1] X^{k-r}\right|_{\mid X=q^{N-1-r}} \\
& =\partial_{q}^{r}\left(\sum_{k=0}^{N-1} X^{k}\right)_{\mid X=q^{N-1-r}} \\
& =\partial_{q}^{r}\left(\frac{X^{N}-1}{X-1}\right)_{\mid X=q^{N-1-r}} .
\end{aligned}
$$

Admettons pour l'instant que 


$$
\partial_{q}^{r}\left(\frac{X^{N}-1}{X-1}\right)=(-1)^{r} \frac{[r] !\left(X^{N}-1\right)}{(X-1)(q X-1) \cdots\left(q^{r} X-1\right)}
$$

Si $q=1$, alors $N$ est un nombre premier sous l'hypothèse $\left(\mathrm{H}_{1}\right)$ et donc

$$
\frac{\left(X^{N-1}\right)}{(X-1)(q X-1) \cdots\left(q^{r} X-1\right)}=\frac{(X-1)^{N}}{(X-1)^{r+1}}=(X-1)^{N-r-1} \text {. }
$$

polynôme qui admet 1 comme racine lorsque $r<N-1$. Par conséquent,

$$
u(r)=(-1)^{r} r !(X-1)^{N-r-1}{ }_{\mid X=1}=0 .
$$

Si $q \neq 1$, alors $q$ est une racine primitive $N$-ième de 1 . ce qui nous permet d'écrire $X^{N}-1$ comme produit des polynômes $\left(X-q^{-i}\right)$ où $i=0, \cdots, N-1$. Il en résulte que

$$
\frac{\left(X^{N}-1\right)}{(X-1)(q X-1) \cdots\left(q^{r} X-1\right)}=q^{-r(r+1) / 2} \prod_{i=r+1}^{N-1}\left(X-q^{-1}\right)
$$

s'annule pour $X=q^{N-1-r}=q^{-(r+1)}$. La nullité de $a(r)$ pour $r<N-1$ en découle.

Il reste à démontrer (5.6). Posons

$$
P_{r}=\partial_{q}^{r}\left(\frac{X^{N}-1}{X-1}\right)
$$

On va établir (5.6) par récurrence sur $r$. C'est vrai pour $r=0$. On sait que l'opérateur $\partial_{q}$ est une $\tau_{q}$-dérivation de $k[X]$ où $\tau_{q}$ est l'automorphisme d'algèbre qui envoie $X$ sur $q X$, c'est-à-dire que l'on a

$$
\partial_{q}\left(P_{1} P_{2} \cdots P_{n}\right)=\sum_{i=1}^{n} \tau_{q}\left(P_{1} \cdots P_{\imath-1}\right) \partial_{q}\left(P_{\imath}\right) P_{\imath+1} \cdots P_{n}
$$

pour tous $P_{1} P_{2}, \cdots P_{n} \in k[X]$. Supposons la formule (5.6) démontrée jusqu'au cran $r$. Appliquons l'opérateur $\partial_{q}$ aux deux membres de l'identité

$$
(-1)^{r}[r] !\left(X^{N}-1\right)=(X-1)(q X-1)\left(q^{r} X-1\right) P_{r}
$$

A gauche, on obtient $(-1)^{r}[r] ![N] X^{N-1}$ qui est nul sous l'hypothèse $\left(\mathrm{H}_{1}\right)$. A droite, la formule de Leibniz (5.7) donne

$$
(q X-1) \cdots\left(q^{r} X-1\right)\left(\left(1+q+\cdots+q^{r}\right) P_{r}+\left(q^{r+1} X-1\right) P_{r+1}\right) .
$$

Par conséquent,

$$
P_{r+1}=-\frac{[r+1] P_{r}}{\left(q^{r+1} X-1\right)}=(-1)^{r+1} \frac{[r+1] !\left(X^{N}-1\right)}{(X-1)(q X-1) \cdots\left(q^{r} X-1\right)\left(q^{r+1} X-1\right)}
$$

ce qu'il fallait démontrer. 
La seconde formule du lemme 5.5 se ramène à la première comme suit. Considérons l'algèbre $\mathscr{D}_{q}^{\prime}$ engendrée par les variables $\tilde{X}$ et $\tilde{Y}$ et la relation $\tilde{Y} \tilde{X}-$ $q^{-1} \tilde{X} \tilde{Y}=1$. Elle est obtenue de $\mathscr{D}_{q}$ en remplacant $q$ par $q^{-1}$. Dans $\mathscr{D}_{q}^{\prime}$ la première formule du lemme 5.5 s'écrit

$$
\sum_{k=0}^{N-1} \tilde{X}^{N-k-1} \tilde{Y}^{N-1} \widetilde{X}^{k}=q^{-(N-1)(N-2) / 2}[N-1] !
$$

Il ne reste plus qu'à lui appliquer le morphisme d'algèbres $\alpha: \mathscr{D}_{q}^{\prime} \rightarrow \mathscr{D}_{q}$ défini par

$$
\alpha(\widetilde{X})=-q Y \text { et } \alpha(\tilde{Y})=X,
$$

ce qui nous donne la seconde formule du lemme.

5.6. Démonstration de la Proposition 5.2. L'assertion $\delta^{N}=0$ résulte du corollaire 5.4 avec $\ell=1$.

Etablissons maintenant l'acyclicité de $(C, \delta)$. Considérons l'application $\sigma$ : $C_{n} \rightarrow C_{n+1}$ définie par $\sigma=q^{-n} s_{n}$. On a

$$
\delta \sigma-q^{-1} \sigma \delta=\mathrm{id}
$$

En effet, les relations de commutation simpliciales donnent

$$
\begin{aligned}
\delta \sigma & =\sum_{i=0}^{n} q^{i-n} d_{\imath} s_{n}=\sum_{i=0}^{n-1} q^{i-n} d_{i} s_{n}+d_{n} s_{n} \\
& =\sum_{i=0}^{n-1} q^{i-n} s_{n-1} d_{i}+\mathrm{id}=q^{-n} s_{n-1}\left(\sum_{i=0}^{n-1} q^{i} d_{i}\right)+\mathrm{id} \\
& =q^{-1} \sigma \delta+\mathrm{id} .
\end{aligned}
$$

Sous l'hypothèse $\left(\mathrm{H}_{1}\right)$ on peut appliquer la première formule du lemme 5.5 avec $X=\delta$ et $Y=-q^{-1} \sigma$, ce qui donne

$$
(-1)^{N-1} q^{-(N-1)} \sum_{k=0}^{N-1} \delta^{N-k-1} \sigma^{N-1} \delta^{k}=[N-1] \text { !id. }
$$

Puisque $[N-1]$ ! est inversible sous nos hypothèses, l'application $\sigma^{N-1}$ convenablement normalisée est une homotopie entre l'identité et l'application nulle.

5.7. Remarque. Le $N$-complexe $C$ muni de la différentielle (5.3) est encore acyclique lorsqu'il existe une application linéaire $s: C_{n} \rightarrow C_{n+1}$ telle que

$$
d_{0} s=\text { id et } d_{i} s=s d_{i-1}
$$

pour tout $i>0$. En effet, dans ce cas on a 


$$
\begin{aligned}
\delta s & =\sum_{i=0}^{n-1} q^{i} d_{i} s+[\ell] q^{n} d_{n} s=d_{0} s+\sum_{i=1}^{n-1} q^{i} d_{i} s+[\ell] q^{n} d_{n} s \\
& =\mathrm{id}+\sum_{i=1}^{n-1} q^{i} s d_{i-1}+[\ell] q^{n} s d_{n-1} \\
& =\mathrm{id}+\sum_{i=0}^{n-2} q^{i+1} s d_{i}+[\ell] q^{n} s d_{n-1}=\mathrm{id}+q s \delta .
\end{aligned}
$$

La relation $\delta s-q s \delta=$ id nous permet d'appliquer la seconde fornule du lemme 5.5 avec $X=s$ et $Y=\delta$, et d'obtenir ainsi l'identité

$$
\sum_{k=0}^{N-1} \delta^{N-k-1} S^{N-1} \delta^{k}=(-1)^{N-1} q^{-N(N-1) / 2}[N-1] \text { !id. }
$$

On conclut comme au $§ 5.6$.

Ceci s'applique, en particulier, au cas $\ell=-1$, où l'on retrouve la différentielle $d_{q}$ considérée dans [1]. De même, le lemme 2 de [1] qui déduit l'acyclicite de $(C, \delta)$ de l'existence d'une application $s$ vérifiant $s \delta-q \delta s=\mathrm{id}$, peut se démontrer à l'aide de la première identité du lemme 5.5 .

5.8. Application. Soit $A$ une algèbre associative unifère et $\left(C(A), d_{\imath}, s_{l}\right)$ le module simplicial de Hochschild de $A$. Rappelons que $C_{n}(A)=A^{\otimes(n+1)}$ et que les applications $d_{i}: C_{n}(A) \rightarrow C_{n-1}(A)$ et $s_{i}: C_{n}(A) \rightarrow C_{n+1}(A)$ sont données pour $a_{0}, a_{1}, \cdots, a_{n} \in A$ et $i=0, \cdots, n$ par

$$
d_{1}\left(a_{0} \otimes a_{1} \otimes \cdots \otimes a_{n}\right)=\left\{\begin{array}{cl}
a_{0} \otimes \cdots \otimes a_{i} a_{i+1} \otimes \cdots \otimes a_{n} & \text { si } 0 \leq i \leq n-1, \\
a_{n} a_{0} \otimes a_{1} \otimes \cdots \otimes a_{n-1} & \text { si } i=n .
\end{array}\right.
$$

et

$$
s_{\imath}\left(a_{0} \otimes a_{1} \otimes \cdots \otimes a_{n}\right)=a_{0} \otimes \cdots \otimes a_{1} \otimes 1 \otimes a_{\imath+1} \otimes \cdots \otimes a_{n}
$$

5.9. Proposition. Soit $B=A \otimes A^{\circ}$ l'algèbre enveloppante de l'algèbre associative unifère $A$. Posons $P_{n}=A^{\otimes(n+2)}$ si $n \geq 0$ et $P_{n}=0$ si $n<0$. On définit $\varepsilon$ : $P_{0} \rightarrow A$ et $d^{\prime}: P_{n} \rightarrow P_{n-1}$ par

$$
\varepsilon\left(a_{0} \otimes a_{1}\right)=a_{0} a_{1} \quad \text { et } d^{\prime}\left(a_{0} \otimes a_{1} \otimes \cdots \otimes a_{n+1}\right)=\sum_{i=0}^{n} q^{1} a_{0} \otimes \cdots \otimes a_{i} a_{i+1} \otimes \cdots \otimes a_{n+1}
$$

où $a_{0}, a_{1}, \cdots, a_{n} \in A$. Alors $\left(P, d^{\prime}, \varepsilon: P_{0} \rightarrow A\right)$ est une $N$-résolution de $A$ pour la classe projective $k$-scindée $\left(\mathscr{P}_{k}, \bigotimes_{k}\right)$ de ${ }_{B} \bmod$.

Démonstration. Les $B$-modules $P_{n}$ sont des modules projectifs relativement aux morphismes $k$-scindés puisqu'ils sont de la forme $A \otimes A^{\otimes n} \otimes A \cong B \otimes A^{\otimes n}$. L'acyclicité du $N$-complexe $\left(P, d^{\prime}, \varepsilon: P_{0} \rightarrow A\right)$ est une conséquence de la 
proposition 5.2 .

5.10. Démonstration du Théorème 1. Considérons la $N$-résolution $\mathscr{P}_{k^{-}}$ projective $\left(P, d^{\prime}, \varepsilon: P_{0} \rightarrow A\right)$ de $B$-modules fournie par la proposition 5.9. Un calcul facile montre que le $N$-complexe $\left(P \otimes{ }_{B} A, d^{\prime} \otimes \mathrm{id}\right)$ est isomorphe à $\left(C_{*}(A), b\right)$, le $N$-complexe $(0.1)$ de l'introduction. Par conséquent, son homologie coincide avec les avatars de l'homologie de Hochschild définis par (0.2):

$$
{ }_{p} H_{*}\left(P \otimes_{B} A, d^{\prime} \bigotimes_{\mathrm{id}}\right) \cong_{p} H H_{*}(A) .
$$

Or. d'après la définition 3.2 appliquée à la même $N$-résolution $\mathscr{P}_{k}$-projective, on a

$$
{ }_{p} H_{*}\left(P \otimes_{B} A, d^{\prime} \otimes \mathrm{id}\right) \cong_{p} \operatorname{Tor}_{*}^{B}(A, A),
$$

d'où nous tirons l'isomorphisme (0.3) de l'introduction. On termine la démonstration du théorème 1 en utilisant le corollaire 3.3 et en rappelant que l'homologie de Hochschild usuelle (c'est-à-dire celle du complexe de Hochschild standard) est isomorphe à $\operatorname{Tor}_{*}^{B}(A, A)$.

5.11. Remarque. Les mêmes méthodes permettent de donner une version cohomologique du théorème 1 . Nous laissons au lecteur le soin de l'énoncer.

\section{Références}

[1] Duhois-Violette, $M$ Generalized differential spaces with $d^{N}=0$ and the $q$-differential calculus, Czechoslovak I. Phys , 46 (1996), 1227-1233.

[2] Dubois-Violette, $M$. et Kerner, R., Universal $q$-differential calculus and $q$-analog of homological algebra. Arta Math Univ. Comenian. (N.S.), 65 (1996). 175-188.

[3] Unversal $Z_{N}$-graded differential calculus, J. Geom. Phys., 23 (1997), 235-246.

[4] Ellenberg. S. et Moore, J.C.. Foundations of relative nomologicul algcbru, Memoirs of the Amer. Math. Soc. 55. Providence, Rhode Island (1965).

[5] Kapranov. M. M.. On the q-analog of homological algebra, preprnt q-alg/9611005.

[6] Maver. W.. A new homology theory, I. II. Annals of Math.. 43 (1942), 370-380 et 594-605.

[7] Sarkarı, K. S.. Combinatorial methnds in topology, Notes of Chandigarh Topology Seminar (1994-95).

[8] - Some simplicial (co) homologies, preprint IHES/M/95/83.

[9] Spanier. E. H., The Mayer homology theory. Bull. Amer. Math. Soc, 55 (1949). 102-112. 